\title{
Regulatory effect of immunosuppressive agents in mice with renal ischemia reperfusion injury
}

\author{
WEI ZHANG ${ }^{1}$, JUNPENG ZHAO ${ }^{2}$, FUYUAN CAO ${ }^{3}$ and SHUMIN LI ${ }^{1}$ \\ ${ }^{1}$ Department of Physiology, School of Basic Medical Science, North China University of Science and Technology; \\ ${ }^{2}$ Clinical Laboratory, Center for Disease Control and Prevention of Tangshan City; ${ }^{3}$ Laboratory Animal Center, \\ North China University of Science and Technology, Tangshan, Hebei 063210, P.R. China
}

Received February 6, 2018; Accepted August 6, 2018

DOI: $10.3892 /$ etm.2018.6642

\begin{abstract}
This study was designed to investigate the regulatory effect of rapamycin in mice with renal ischemia-reperfusion injury. A total of 100 mice were randomly divided into normal control, sham operation, model and experimental groups with 25 rats in each group. Mice in the experimental group were subjected to rapamycin gavage. Mice in each group were sacrificed $24 \mathrm{~h}$ after operation. Then, blood, spleen and left kidney were collected. PAS staining was used for semi-quantitative analysis of renal pathological injury. Serum creatinine ( $\mathrm{SCr}$ ) and blood urea nitrogen (BUN) levels were measured. TUNEL method was used to detect cell apoptosis. Flow cytometry was used to detect the percentage of NKT cells. The expression of CXC chemokine ligand 10 (CXCL10), hypoxia-inducible factor-1 $\alpha$ (HIF-1 $\alpha$ ) and vascular endothelial growth factor (VEGF) mRNA were detected by RT-qPCR. Semi-quantitative scoring of renal pathological injury showed that pathological injury score of the experimental group was significantly lower than that of the model group $(\mathrm{p}<0.05)$. Serum levels of $\mathrm{SCr}$ and BUN in the experimental group was compared to those in the model group $(\mathrm{p}<0.05)$. The number of apoptotic cells in the experimental group was compared to that of the model group $(\mathrm{p}<0.05)$. The percentage of NKT cells in the experimental group was compared to that of the model group $(\mathrm{p}<0.05)$. The percentage of NKT cells was significantly higher in the kidney and peripheral blood of the experimental group than that in the model group $(\mathrm{p}<0.05)$. The expression levels of HIF- $1 \alpha$ and VEGF mRNA in the model and experimental groups were significantly lower in the experimental group than those in the model group $(\mathrm{p}<0.05)$. The expression levels
\end{abstract}

Correspondence to: Dr Shumin Li, Department of Physiology, School of Basic Medical Science, North China University of Science and Technology, 21 Bohai Avenue, Tangshan, Hebei 063210, P.R. China

E-mail: ssj25h@163.com

Key words: renal ischemia-reperfusion injury, rapamycin, natural killer cells, hypoxia-inducible factor- $1 \alpha, \mathrm{CXC}$ chemokine ligand 10 of CXCL10 mRNA in the model and experimental groups were significantly higher in the experimental group than those in the model group $(\mathrm{p}<0.05)$. The results indicated that rapamycin can significantly upregulate the expression level of CXCL9 and promote the accumulation of NKT cells in kidney from spleen through peripheral blood. Rapamycin can also inhibit the HIF-1 $\alpha$ expression level and protect renal ischemia-reperfusion injury.

\section{Introduction}

Temporary blocking of renal blood flow is required in some complex renal surgeries, such as renal parenchymal lithotomy, renal transplantation and renal tumor resection. Postoperative reperfusion may lead to increased injury of ische mic renal tissue, which is called renal ischemia-reperfusion injury (RIRI) (1). RIRI can lead to renal failure and acute renal insufficiency, causing high morbidity and mortality. A study has shown that the mechanism of RIRI is very complicated, and many internal factors, such as vascular obstruction, inflammatory mediators, enhanced acidic environment interstitial edema, as well as calcium and oxygen-free radical damage, were involved (2). A study showed that adaptive immune system and innate immunity play an important role in RIRI. Immune system intervention may have a protective effect on RIRI (3). Rapamycin, as a novel immunosuppressive agent, can bind mammalian target of rapamycin (mTOR) via receptor FK50-binding protein-12 to inhibit its signal transduction, thereby reducing the arrest of immune cells in late G1 and suppressing immune response (4). The protective mechanism of rapamycin on ischemia-reperfusion injury is still unclear. In view of this, our study investigated the protective effects of rapamycin on RIRI, and explored the mechanism, so as to find a way to reduce renal ischemia-reperfusion injury.

\section{Materials and methods}

Experimental animals and grouping. A total of 100 SPF C57BL/6 male mice weighing 20-25 g (8 weeks old) were purchased from Shanghai Laboratory Animal Center, Chinese Academy of Sciences (Shanghai, China). The mice were raised in cages at $20-25^{\circ} \mathrm{C}$, with free access to food and water. Mice 
were divided randomly into the normal control, sham operation, model and experimental groups with 25 mice in each group. The study was approved by the Ethics Committee of North China University of Science and Technology (Tangshan, China).

Main reagents. Rapamycin was from Wyeth: Pfizer (Collegeville, PA, USA); TUNEL Apoptosis Detection kit was purchased from Wuhan Boster Biological Technology, Ltd. (Wuhan, China); RT-qPCR kit was purchased from Takara Biotechnology Co., Ltd. (Dalian, China); rat anti-mouse NK1.1 monoclonal antibody was purchased from R\&D Systems, Inc. (1:300; cat. no. 694370, Minneapolis, MN, USA).

Main equipment. Ultra-clean workbench (AIRTECH; Suzhou Purification Equipment Co., Ltd., Suzhou, China); precision electronic balance (Mettler-Toledo GmbH, Greifensee, Switzerland); UV spectrophotometer (Shanghai Third Analytical Instrument Factory, Shanghai, China); PCR instrument (Bio-Rad Laboratories, Inc., Hercules, CA, USA); Cytomics $^{\mathrm{TM}}$ FC 500 series flow cytometer and AU680 automatic biochemical analyzer (both from Beckman Coulter, Inc., Brea, CA, USA).

Animal model construction. RIRI model was constructed according to the methods described in a previous study (5). Mice were fasted for $12 \mathrm{~h}$ before operation but were allowed to drink water. After anesthesia (injected with $10 \%$ chloral hydrate), the mice were fixed in supine position. Skin was disinfected with $75 \%$ ethanol, and an incision was made along the middle line of the abdomen. Left renal pedicle was separated and clipped with non-invasive microvascular clip. Kidneys gradually changed from bright red to dark purple. Clips were removed 30 min later, and the rapid recovery of kidney color indicated that the model was successfully established. The skin was closed and incision was coated with bupivacaine for analgesia after operation. Bilateral renal pedicle was not clipped in the sham operation group and other steps were the same as the model group. Mice in the control group were not treated.

Animal treatment. Rapamycin (3 $\mathrm{mg} / \mathrm{kg} /$ day) gavage was performed to mice in the experimental group 2 days and 1 day before surgical operation and after successful establishment of the model. Mice in each group were sacrificed at $24 \mathrm{~h}$ after operation, and blood, spleen and left kidney were collected. The left kidney was cut into two halves, one half was fixed in formaldehyde, and the other half was stored in liquid nitrogen for RT-qPCR detection.

Semi-quantitative analysis of renal pathological injury. After fixation, embedding, slicing and PAS staining, semi-quantitative analysis of renal pathological injury was performed. Ten visual fields of renal plexus junction were randomly selected under high magnification microscope (Olympus, Tokyo, Japan) (x400) to observe the degree of injury. Scoring standards were: 0 for normal, lesion $<25 \%$ for 1 point, $25-50 \%$ for 2 points, $51-75 \%$ for 3 points, and $>75 \%$ for 4 points.

Renal function. Serum creatinine ( $\mathrm{SCr}$ ) and blood urea nitrogen (BUN) were detected using Beckman Coulter AU680 automatic biochemical analyzer.
Cell apoptosis test. Kidneys were fixed overnight in 4\% paraformaldehyde solution, and embedded in paraffin. TUNEL method was used to detect cell apoptosis according to the instructions. Red fluorescence under microscope (Olympus) indicated TUNEL-positive cells. Six non-repetitive visual fields (x200) were randomly selected to calculate the positive apoptotic index (AI) according to the formula: $\mathrm{AI}=$ (apoptotic cells/total cells) $\times 100 \%$

Flow cytometry. Blood, spleen and kidney tissues of mice were used to make single cell suspension. After incubation with rat anti-mouse CD3 and NK1.1 monoclonal antibodies (1:300; cat. nos. 17A 2 and $694370, \mathrm{R} \& D$ Systems, Inc., at $4^{\circ} \mathrm{C}$ for $1 \mathrm{~h}$, flow cytometry was used to detect the ratio of NKT cells. CD3 and NK1.1 double-positive cells were defined as NKT cells.

RT-qPCR detection of expression levels of CXC chemokine ligand 10 (CXCL10), hypoxia-inducible factor-1 $\alpha(H I F-1 \alpha)$ and vascular endothelial growth factor (VEGF) $m R N A$. Total RNA was extracted from kidneys using TRIzol. After purification, RNA samples were used in reverse transcription to synthesize cDNA. Primers used in $\mathrm{PCR}$ reactions were: $\beta$-actin forward, 5'-AGGCATCCTGACCCTGAAGTA-3' and reverse, 5'-GAGGCATACAGGGACAACACAG-3'; CXCL10 forward, 5'-ACTGCATCCATATCGATGAC-3' and reverse, 5'-TTC ATCCTGCAATGATCTC-3'; HIF-1 $\alpha$ forward, 5'-AAGTCT AGGGATGCAGCAC-3' and reverse, 5'-CAAGATCACCAG CATCTAG-3'; VEGF forward, 5'-ATTGAGACCCTGGTG GACATC-3' and reverse, 5'-TCCTTTCCTCGAACTGATT-3'. Expression level of each gene was normalized to endogenous control $\beta$-actin using $2^{-\Delta \Delta \mathrm{Cq}}$ method (6).

Statistical analysis. Statistical analyses were performed using SPSS 18.0. (SPSS, Inc., Chicago, IL, USA). Count data were processed by $\chi^{2}$ test. Measurement data were expressed as $(\overline{\mathrm{x}} \pm \mathrm{SD})$. ANOVA was used for comparison between multiple groups and the post hoc test was SNK test. were processed using parallel t-test. $\mathrm{P}<0.05$ was considered to be statistically significant.

\section{Results}

Semi-quantitative analysis of renal pathological injury. Clear renal tubule and glomeruli structure, and no obvious pathological changes were found in the control group. In the model group, renal tubular structure was damaged, epithelial cells were exfoliated and necrotic, basement membrane was exposed, and vacuolar degeneration was also observed. Only a small number of epithelial cells were exfoliated in the experimental group, and proximal renal tubule swelling was also observed. Kidney injury was significantly lower in the experimental group than in the model group. Renal injury score was higher in the model and experimental groups than in the sham operation group $(\mathrm{p}<0.05)$, and the score of the experimental group was significantly lower than that of the model group $(\mathrm{p}<0.05)$ (Fig. 1).

Renal function. Serum levels of SCr and BUN in the model and experimental groups were significantly higher than those 


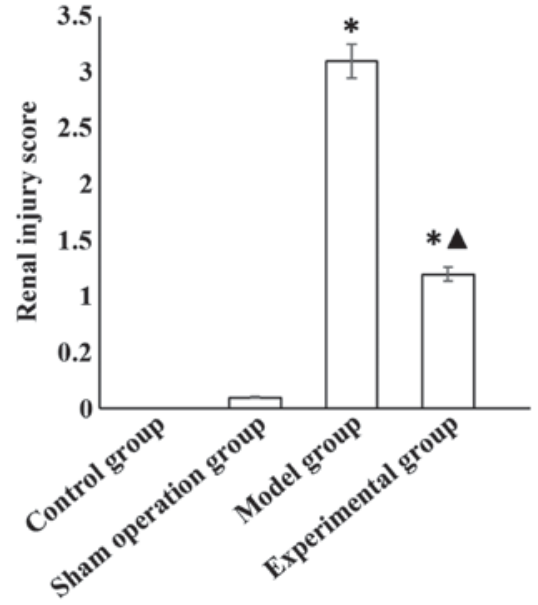

Figure 1. Semi-quantitative analysis of renal pathological injury in mice of each group. ${ }^{*} \mathrm{P}<0.05$, compared with the sham operation group; ${ }^{\wedge} \mathrm{p}<0.05$, compared with the model group.

Table I. Comparison of serum levels of SCr and BUN among groups $(\overline{\mathrm{x}} \pm \mathrm{SD})$.

\begin{tabular}{llc}
\hline Groups & $\mathrm{SCr}(\mu \mathrm{mol} / \mathrm{l})$ & $\mathrm{BUN}(\mathrm{mmol} / \mathrm{l})$ \\
\hline Control & $18.56 \pm 5.26$ & $13.02 \pm 3.01$ \\
Sham operation & $20.12 \pm 4.35$ & $14.58 \pm 2.98$ \\
Model & $62.19 \pm 8.38^{\mathrm{a}}$ & $39.09 \pm 4.36^{\mathrm{a}}$ \\
Experimental & $39.85 \pm 7.45^{\mathrm{a}, \mathrm{b}}$ & $25.35 \pm 3.86^{\mathrm{a}, \mathrm{b}}$ \\
\hline
\end{tabular}

${ }^{\mathrm{a}} \mathrm{P}<0.05$, compared with the sham operation group; ${ }^{\mathrm{b}} \mathrm{p}<0.05$, compared with the model group. SCr, serum creatinine; BUN, blood urea nitrogen.

in the sham operation group $(\mathrm{p}<0.05)$, and were significantly lower in the experimental group than those in the model group $(\mathrm{p}<0.05)$ (Table I).

Cell apoptosis. The number of apoptotic cells in the model and experimental groups was significantly higher than that in sham operation group $(\mathrm{p}<0.05)$, and was significantly lower in the experimental than in the model group $(\mathrm{p}<0.05)$ (Fig. 2).

Percentage of NKT cells. Percentage of NKT cells in spleen of the model and experimental groups was significantly lower than that in the sham operation group $(\mathrm{p}<0.05)$, and was significantly lower in the experimental than that in the model group $(\mathrm{p}<0.05)$. Percentage of NKT cells in kidney and periphe ral blood of the model and experimental groups was significantly higher than that in the sham operation group $(\mathrm{p}<0.05)$, and was significantly higher in the experimental than that in the model group ( $\mathrm{p}<0.05)$ (Fig. 3).

Expression levels of CXCL10, HIF-1 $\alpha$ and VEGF $m R N A$ in different groups. The expression levels of HIF-1 $\alpha$ and VEGF mRNAs in the model and experimental groups were significantly higher than those in the sham-operated group $(\mathrm{p}<0.05)$, and were significantly lower in the experimental group than those in the model group $(\mathrm{p}<0.05)$. The expression level of

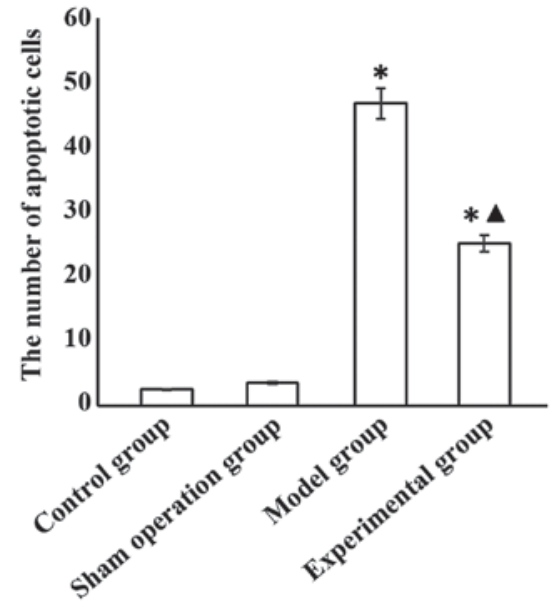

Figure 2. Cell apoptosis in each group. ${ }^{*} \mathrm{P}<0.05$, compared with the sham operation group; ${ }^{\wedge} \mathrm{p}<0.05$, compared with the model group.

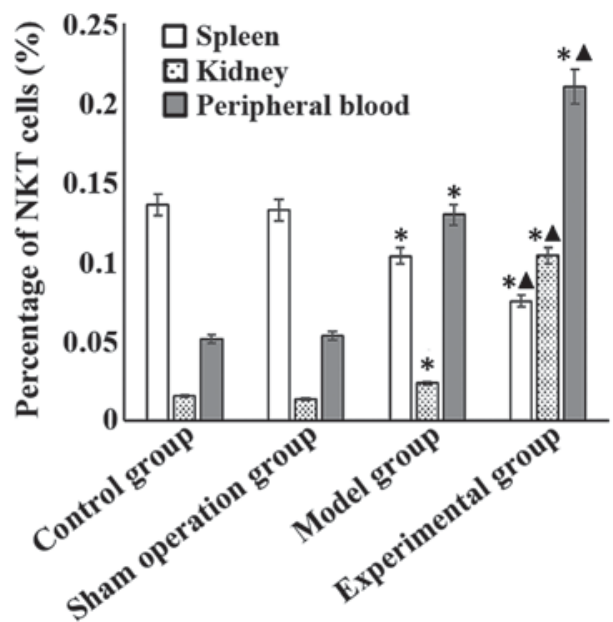

Figure 3. Percentage of NKT cells in spleen, kidney and peripheral blood of mice in each group. " $\mathrm{P}<0.05$, compared with the sham operation group; ${ }^{\star} \mathrm{p}<0.05$, compared with the model group.

CXCL10 mRNA in the model and experimental groups was significantly lower than that in the sham operation group $(\mathrm{p}<0.05)$, and was significantly higher in the experimental group than that in the model group $(\mathrm{p}<0.05)$ (Table II).

\section{Discussion}

As a hyperperfusion organ, kidney is sensitive to ischemia reperfusion. Pathogenesis of RIRI is very complex, and the factors which affect RIRI can interact with each other. So the mechanism of RIRI is still unclear. How to reduce RIRI is now a hot research field. As a novel immunosuppressive agent, the effects of rapamycin on kidney transplantation have been confirmed (7). However, the mechanism of the protective effects of rapamycin on ischemia-reperfusion injury remains controversial.

Results of this study showed that rapamycin could significantly reduce the levels of $\mathrm{SCr}$ and $\mathrm{BUN}$ after renal ischemia-reperfusion in mice to improve renal function, so as to reduce the rate of tubular apoptosis. Histopathological examination also confirmed that rapamycin has a protective 
Table II. Expression levels of CXCL10, HIF-1 $\alpha$ and VEGF mRNA in the different groups ( $\overline{\mathrm{x}} \pm \mathrm{SD})$.

\begin{tabular}{llll}
\hline Groups & \multicolumn{1}{c}{ CXCL10 } & \multicolumn{1}{c}{ HIF-1 $\alpha$} & VEGF \\
\hline Control & $0.3589 \pm 0.040$ & $0.0107 \pm 0.0005$ & $0.0143 \pm 0.0012$ \\
Sham operation & $0.3615 \pm 0.039$ & $0.0106 \pm 0.006$ & $0.0146 \pm 0.0011$ \\
Model & $0.2315 \pm 0.041^{\mathrm{a}}$ & $0.0156 \pm 0.007^{\mathrm{a}}$ & $0.0198 \pm 0.0013^{\mathrm{a}}$ \\
Experimental & $0.2935 \pm 0.038^{\mathrm{a}, \mathrm{b}}$ & $0.0123 \pm 0.008^{\mathrm{a}, \mathrm{b}}$ & $0.0163 \pm 0.0014^{\mathrm{a}, \mathrm{b}}$ \\
\hline
\end{tabular}

${ }^{\mathrm{a}} \mathrm{P}<0.05$, compared with the sham operation group; ${ }^{\mathrm{b}} \mathrm{p}<0.05$, compared with the model group. CXCL10, CXC chemokine ligand $10 ; \mathrm{HIF}-1 \alpha$, hypoxia-inducible factor- $1 \alpha$; VEGF, vascular endothelial growth factor.

effect on RIRI. Khan et al (8) reported that rapamycin had a protective effect on liver ischemia reperfusion injury in mice, which is consistent with the results of our study. Lui et al (9) reported that in the early stage of RIRI, rapamycin could reduce the degree of renal damage through its anti-apoptotic effect, and in the late stage of RIRI, rapamycin could aggravate ischemia-reperfusion injury by inhibiting tissue repair. Ischemia-reperfusion injury refers to the increased degree of ischemic tissue injury after reperfusion. Rapamycin is a macrolide antibiotic isolated from the culture medium of actinomycetes. Besides the application in the treatment of fungal infections and tumors, rapamycin has also been used in organ transplantation (10). Rapamycin blocks various signaling pathways through different cytokine receptors and blocks the progression of lymphocytes from the $\mathrm{G} 1$ phase to the $\mathrm{S}$ phase, thereby producing an immunosuppressive effect $(11,12)$.

NKT cell is a kind of immune cell. In recent years, studies on NKT cell have attracted increasing attention. A previous study showed that NKT cells play an important role in ischemia-reperfusion injury, in which NKT cells can be recruited from spleen through peripheral blood to target organ damage (13). Results of our study showed that the percentage of NKT cells in spleen of the model and experimental groups was significantly lower than that in the sham operation group $(p<0.05)$, and the percentage was also significantly lower in the experimental group than that in the model group $(\mathrm{p}<0.05)$. The percentage of NKT cells in kidney and peripheral blood of the model and experimental groups was significantly higher than that in the sham operation group $(\mathrm{p}<0.05)$, and the percentage was also significantly higher in the experimental group than that in the model group $(\mathrm{p}<0.05)$, indicating that rapamycin can promote moving of NKT cells from spleen through the peripheral blood to kidney, leading to the higher percentage of NKT cells in kidney after ischemia-reperfusion injury, so as to protect the kidney. This may be one of the mechanisms by which rapamycin exerts a protective effect on RIRI.

HIF- $1 \alpha$ is a cytokine that is widely distributed in various tissues and is highly sensitive to the concentration of oxygen. Under normoxic conditions, HIF-1 $\alpha$ gene is continuously transcribed and translated, but is also degraded by ubiquitous degradation, so the level of HIF-1 $\alpha$ in the body is very low (14). Under hypoxic conditions, the degradation of HIF-1 $\alpha$ was inhibited and the level of HIF-1 $\alpha$ was increased. HIF-1 $\alpha$ can form a dimer with HIF-1 $\beta$ to upregulate VEGF expression, promote angiogenesis, and improve oxygen supply, so as to make tissues adapt to hypoxia (15). Our data showed that the expression levels of HIF-1 $\alpha$ and VEGF mRNAs in the model and experimental groups were significantly higher than those in the sham operation group $(\mathrm{p}<0.05)$, and the expression levels in the experimental group was significantly lower than those in the model group $(\mathrm{p}<0.05)$, indicating that rapamycin may have an anti-apoptotic effect by inhibiting the expression of HIF-1 $\alpha$. To date, $>50$ chemokines have been identified, among which chemokine CXC is a group of cytokines closely related to angiogenesis. CXC can be divided into ELR and non-ELR chemokines. The former has the function of promoting angiogenesis, while the latter has the effect on inhibiting angiogenesis (10). Our data show that the CXCL10 mRNA expression level in the model and experimental groups was significantly lower than that in the sham operation group $(p<0.05)$, and the expression level in the experimental group was significantly higher than that in the model group $(\mathrm{p}<0.05)$, indicating that rapamycin can significantly upregulate the expression of CXCL9, which may be involved in the recruitment of NKT cells to kidney after ischemia-reperfusion injury (16).

In summary, rapamycin can significantly upregulate the expression of CXCL9 and promote recruitment of NKT cells from spleen through peripheral blood to kidney. Rapamycin can also inhibit the expression of HIF- $1 \alpha$, so as to have a protective effect on RIRI.

\section{Acknowledgements}

Not applicable.

\section{Funding}

No funding was received.

\section{Availability of data and materials}

The datasets used and/or analyzed during the present study are available from the corresponding author on reasonable request.

\section{Authors' contributions}

WZ and SL conceived and designed the study. JZ collected the data. FC was responsible for the analysis and interpretation of the data. WZ drafted this manuscript. SL revised the manuscript critically for important intellectual content. All authors have read and approved the final manuscript. 


\section{Ethics approval and consent to participate}

The study was approved by the Ethics Committee of North China University of Science and Technology (Tangshan, China).

\section{Patient consent for publication}

Not applicable.

\section{Competing interests}

The authors declare that they have no competing interests.

\section{References}

1. Zandstra J, van Beuge MM, Zuidema J, Petersen AH, Staal M, Duque LF, Rodriguez S, Lathuile AA, Veldhuis GJ, Steendam R, et al: Microsphere-based rapamycin delivery, systemic versus local administration in a rat model of renal ischemia/reperfusion injury. Pharm Res 32: 3238-3247, 2015.

2. Malek M and Nematbakhsh M: Renal ischemia/reperfusion injury; from pathophysiology to treatment. J Renal Inj Prev 4: 20-27, 2015.

3. Kezić A, Stajic N and Thaiss F: Innate immune response in kidney ischemia/reperfusion injury: Potential target for therapy. J Immunol Res 2017: 6305439, 2017

4. Zhu J, Lu T, Yue S, Shen X, Gao F, Busuttil RW, Kupiec-Weglinski JW, Xia Q and Zhai Y: Rapamycin protection of livers from ischemia and reperfusion injury is dependent on both autophagy induction and mammalian target of rapamycin complex 2-Akt activation. Transplantation 99: 48-55, 2015

5. Feldman ME, Apsel B, Uotila A, Loewith R, Knight ZA, Ruggero D and Shokat KM: Active-site inhibitors of mTOR target rapamycin-resistant outputs of $\mathrm{mTORC} 1$ and $\mathrm{mTORC} 2$. PLoS Biol 7: e38, 2009.

6. Livak KJ and Schmittgen TD: Analysis of relative gene expression data using realtime quantitative PCR and the 2(-Delta Delta C(T)) method. Methods 25: 402-408, 2001.
7. Guan X, Qian Y, Shen Y, Zhang L, Du Y, Dai H, Qian J and Yan Y: Autophagy protects renal tubular cells against ischemia/reperfusion injury in a time-dependent manner. Cell Physiol Biochem 36: 285-298, 2015.

8. Khan S, Salloum F, Das A, Xi L, Vetrovec GW and Kukreja RC: Rapamycin confers preconditioning-like protection against ischemia-reperfusion injury in isolated mouse heart and cardiomyocytes. J Mol Cell Cardiol 41: 256-264, 2006.

9. Lui SL, Chan KW, Tsang R, Yung S, Lai KN and Chan TM: Effect of rapamycin on renal ischemia-reperfusion injury in mice. Transpl Int 19: 834-839, 2006.

10. Connell BJ, Gordon JR and Saleh TM: ELR-CXC chemokine antagonism is neuroprotective in a rat model of ischemic stroke. Neurosci Lett 606: 117-122, 2015.

11. Baroja-Mazo A, Revilla-Nuin B, Ramírez P and Pons JA: Immunosuppressive potency of mechanistic target of rapamycin inhibitors in solid-organ transplantation. World J Transplant 6: 183-192, 2016.

12. Zhang Y, He S, Du X, Jiang Y, Tian B and Xu S: Rapamycin suppresses hypoxia/reoxygenation-induced islet injury by up-regulation of miR-21 via PI3K/Akt signalling pathway. Cell Prolif 50: 1-8, 2017.

13. Zimmerman MA, Martin A, Yee J, Schiller J and Hong JC: Natural killer T cells in liver ischemia-reperfusion injury. J Clin Med 6: 41, 2017.

14. Hagen T: Oxygen versus reactive oxygen in the regulation of HIF-1 $\alpha$ : The balance tips. Biochem Res Int 2012: 436981, 2012.

15. Coothankandaswamy V, Liu Y, Mao SC, Morgan JB, Mahdi F, Jekabsons MB, Nagle DG and Zhou YD: The alternative medicine pawpaw and its acetogenin constituents suppress tumor angiogenesis via the HIF-1/VEGF pathway. J Nat Prod 73: 956-961, 2010.

16. Javedan G, Shidfar F, Davoodi SH, Ajami M, Gorjipour F, Sureda A, Nabavi SM, Daglia M and Pazoki-Toroudi H: Conjugated linoleic acid rat pretreatment reduces renal damage in ischemia/reperfusion injury: Unraveling antiapoptotic mechanisms and regulation of phosphorylated mammalian target of rapamycin. Mol Nutr Food Res 60: 2665-2677, 2016.

(i) $\ominus$ This work is licensed under a Creative Commons Attribution-NonCommercial-NoDerivatives 4.0 International (CC BY-NC-ND 4.0) License. 